\title{
A SELECTION THEOREM AND ITS APPLICATIONS
}

\author{
Xie Ping Ding, Won Kyu Kim and KoK-Keong Tan
}

In this paper, we first prove an improved version of the selection theorem of Yannelis-Prabhakar and next prove a fixed point theorem in a non-compact product space. As applications, an intersection theorem and two equilibrium existence theorems for a non-compact abstract economy are given.

\section{INTRODUCTION}

In convex analysis, the Fan-Browder fixed point theorem [2] is an essential tool in proving existence theorems of numerous nonlinear problems (for example see [2, 7, 13, 15]). Actually, the Fan-Browder fixed point theorem can be proved by constructing a continuous selection.

In [15], Yannelis-Prabhakar proved a continuous selection theorem and obtained a fixed point theorem in paracompact convex sets. Using this fixed point theorem, they obtained an equilibrium existence theorem for a compact abstract economy.

In this paper, we first give an improved version of the selection theorem of YannelisPrabhakar [15]. By applying this result, we prove a fixed point theorem in non-compact product spaces. As an application of our fixed point theorem, we first prove an intersection theorem which is closely related to a non-compact generalisation of Fan's intersection theorem [6] due to Shih-Tan [12]. Next, two equilibrium existence theorems are obtained which are either closely related to or generalisations of those results of Borglin-Keiding [1], Shafer-Sonnenschein [11], Tarafdar [14] and Yannelis-Prabhakar [15].

We shall need the following notations and definitions. Let $A$ be a non-empty set. We shall denote by $2^{A}$ the family of all subsets of $A$. If $A$ is a non-empty subset of a topological space $X$, we shall denote by $c_{X} A$ the closure of $A$ in $X$. If $A$ is a subset of a vector space, co $A$ denotes the convex hull of $A$. Let $X, Y$ be topological spaces and $\phi: X \rightarrow 2^{Y}$ be a correspondence.

(i) If $A \subset X$, we shall denote the restriction of $\phi$ to $A$ by $\left.\phi\right|_{A}$, that is, $\left.\phi\right|_{A}: A \rightarrow 2^{Y}$ is the correspondence defined by $\left.\phi\right|_{A}(x)=\phi(x)$ for all $\boldsymbol{x} \in \boldsymbol{A}$.

\section{Received 4th September, 1991.}

This paper was partially supported by NSERC of Canada under grant A-8096, and for the second author by a grant from the Korea Science and Engineering Foundation in 1992.

Copyright Clearance Centre, Inc. Serial-fee code: 0004-9729/92 \$A2.00+0.00. 
(ii) $\phi$ is said to be upper semicontinuous if for each open subset $V$ of $Y$, the set $\{x \in X: \phi(x) \subset V\}$ is open in $X$.

(iii) $f: X \rightarrow Y$ is a continuous selection of $\phi$ if $f$ is continuous and $f(x) \in$ $\phi(x)$ for all $x \in X$.

(iv) If $Y$ is a vector space, the correspondence $\operatorname{co} \phi: X \rightarrow 2^{Y}$ is defined by $(\operatorname{co} \phi)(x)=\operatorname{co} \phi(x)$ for all $x \in X$.

\section{Selection AND FIXED POINT TheOREMS}

We shall first generalise a selection theorem of Yannelis-Prabhakar [15, Theorem 3.1] as follows :

THEOREM 1. Let $X$ be a non-empty paracompact Hausdorff topological space and $Y$ be a non-empty convex subset of a topological vector space. Suppose $S, T$ : $X \rightarrow 2^{Y}$ are correspondences such that

(1) for each $x \in X, \quad \operatorname{co} S(x) \subset T(x)$ and $S(x) \neq \emptyset$,

(2) for each $y \in Y, S^{-1}(y)$ is open in $X$.

Then $T$ has a continuous selection.

Proof: By (1), $X=\bigcup_{y \in Y} S^{-1}(y)$. Since $X$ is paracompact, by (2) and Lemma 1 of Michael [10], there exists an open locally finite refinement $\mathcal{F}=\left\{U_{a}: a \in A\right\}$ of the family $\left\{S^{-1}(y): y \in Y\right\}$ where $A$ is an index set and $U_{a}$ is an open subset of $X$. By Proposition 2 of Michael [10], there exists a family of continuous functions $\left\{g_{a}: a \in A\right\}$ such that $g_{a}: X \rightarrow[0,1], g_{a}(x)=0$ for $x \notin U_{a}$ and $\sum_{a \in A} g_{a}(x)=1$ for all $x \in X$. For each $a \in A$, choose $y_{a} \in Y$ such that $U_{a} \subset S^{-1}\left(y_{a}\right)$. This can be done since $\mathcal{F}$ is a refinement of $\left\{S^{-1}(y): y \in Y\right\}$. Define $f: X \rightarrow Y$ by

$$
f(x)=\sum_{a \in A} g_{a}(x) y_{a} \quad \text { for each } x \in X
$$

From the local finiteness of $\mathcal{F}$, it follows that for each $x \in X$, at least one, and at most finitely many, $g_{a}(x)$ is not zero, and $f$ is a well-defined continuous function from $X$ to $Y$. Let $x \in X$ and $a \in A$ be such that $g_{a}(x) \neq 0$, then $x \in U_{a} \subset S^{-1}\left(y_{a}\right)$ so that $y_{a} \in S(x)$. By (1) and the definition of $f$, we have $f(x) \in \operatorname{co} S(x) \subset T(x)$ for each $x \in X$. This completes the proof.

If $S=T$, Theorem 1 reduces to Theorem 3.1 of Yannelis-Prabhakar [15].

We shall need the following lemma.

LEMMA 1. Let $D$ be a non-empty compact subset of a topological vector space $E$. Then $\operatorname{co} D$ is $\sigma$-compact and hence is paracompact. 
Proof: The proof that $c o D$ is $\sigma$-compact can be found in [9, p.49]. For completeness, we shall include the simple proof here. For each $n \in N$, let $S_{n}=\left\{\left(\lambda_{1}, \ldots, \lambda_{n}\right)\right.$ : $\lambda_{1}, \ldots, \lambda_{n} \geqslant 0$ with $\left.\sum_{i=1}^{n} \lambda_{i}=1\right\}$ and define $f_{n}: S_{n} \times \prod_{i=1}^{n} D \rightarrow E$ by

$$
f_{n}\left(\lambda_{1}, \ldots, \lambda_{n}, x_{1}, \ldots, x_{n}\right)=\sum_{i=1}^{n} \lambda_{i} x_{i} .
$$

Then $f_{n}$ is continuous. Since $S_{n} \times \prod_{i=1}^{n} D$ is compact, $f_{n}\left(S_{n} \times \prod_{i=1}^{n} D\right)$ is compact. But then $\operatorname{co} D=\bigcup_{n=1}^{\infty} f_{n}\left(S_{n} \times \prod_{i=1}^{n} D\right)$ is $\sigma$-compact. It follows that $\operatorname{co} D$ is Lindelöf. Since co $D$ is regular, co $D$ is paracompact by Corollary 33.15 in [3, p.341]. This completes the proof.

We remark here that the topological vector space $E$ in the above lemma is not assumed to be Hausdorff.

We shall prove the following fixed point theorem.

Theorem 2. Let $\left\{X_{i}\right\}_{i \in I}$ be a family of non-empty convex sets, each in a locally convex Hausdorff topological vector space $E_{i}$, where $I$ is an index set. For each $i \in I$, let $D_{i}$ be a non-empty compact subset of $X_{i}$ and $S_{i}, T_{i}: X=\prod_{i \in I} X_{i} \rightarrow 2^{D_{i}}$ be such that for each $i \in I$,

(1) for each $x \in X$, co $S_{i}(x) \subset T_{i}(x)$ and $S_{i}(x) \neq 0$,

(2) for each $y_{i} \in D_{i}, S_{i}^{-1}\left(y_{i}\right)$ is open in $X$.

Then there exists a point $\widehat{x} \in D=\prod_{i \in I} D_{i}$ such that $\widehat{x} \in T(\widehat{x})=\prod_{i \in I} T_{i}(\widehat{x})$, that is, $\widehat{x}_{i} \in T_{i}(\widehat{x})$ for all $i \in I$, where $\widehat{x}_{i}$ is the projection of $\widehat{x}$ onto $X_{i}$ for each $i \in I$.

Proof: Since $D=\prod_{i \in I} D_{i}$ is compact in $X$, it follows from Lemma 1 that co $D$ is paracompact in $X$. For each $i \in I$, let $S_{i}^{*}, T_{i}^{*}$ be the restrictions of $S_{i}, T_{i}$ on co $D$, then we have

(a) for each $x \in \operatorname{co} D, \quad \operatorname{co} S_{i}^{*}(x) \subset \operatorname{co} T_{i}^{*}(x)$ and $\operatorname{co} S_{i}^{*}(x) \neq \emptyset$,

(b) for each $y_{i} \in D_{i}$,

$$
\begin{aligned}
\left(S_{i}^{*}\right)^{-1}\left(y_{i}\right) & =\left\{x \in \operatorname{co} D: y_{i} \in S_{i}^{*}(x)\right\} \\
& =\left\{x \in \operatorname{co} D: y_{i} \in S_{i}(x)\right\} \\
& =\operatorname{co} D \cap S_{i}^{-1}\left(y_{i}\right)
\end{aligned}
$$

is open in $\operatorname{co} D$.

By Theorem 1, for each $i \in I, T_{i}^{*}$ has a continuous selection $f_{i}: \operatorname{co} D \rightarrow D_{i}$ such that $f_{i}(x) \in T_{i}^{*}(x)=T_{i}(x)$ for each $x \in \operatorname{co} D$. 
Define $f: \operatorname{co} D \rightarrow D$ and $T: \operatorname{co} D \rightarrow 2^{D}$ by

$$
f(x)=\prod_{i \in I} f_{i}(x) \quad \text { and } \quad T(x)=\prod_{i \in I} T_{i}(x) \quad \text { for each } x \in \operatorname{coD} .
$$

Then $f$ is clearly continuous. By Theorem 4.5.1 of Smart [13], there exists $\widehat{x} \in D$ such that $\widehat{x}=f(\widehat{x}) \in T(\widehat{x})$. This completes the proof.

Theorem 2 generalises Theorem 3.2 of Yannelis-Prabhakar [15] in several ways :

(i) I need not be a singleton set,

(ii) $X_{i}$ need not be paracompact, and

(iii) $S_{i}$ and $T_{i}$ need not be identical.

\section{Applications}

Let $X_{1}, \ldots, X_{n}(n \geqslant 2)$ be topological spaces and $X=\prod_{i=1}^{n} X_{i}$. Let $i \in\{1, \ldots, n\}$ be arbitrarily fixed. Let $\widehat{X}_{i}=\prod_{\substack{j=1 \\ j \neq i}}^{n} X_{j}$ and $\pi_{i}: X \rightarrow X_{i}$ and $\widehat{\pi}_{i}: X \rightarrow \widehat{X}_{i}$ be the projections. If $x \in X$, we can write $\pi_{i}(x)=x_{i}$ and $\widehat{\pi}_{i}(x)=\widehat{x}_{i}$. Let $A$ be a subset of $X, x_{i} \in X_{i}$ and $\widehat{x}_{i} \in \widehat{X}_{i}$. Then $\left[x_{i}, \widehat{x}_{i}\right]$ denotes the point $x \in X$ such that $\pi_{i}(x)=x_{i}$ and $\widehat{\pi}_{i}(x)=\widehat{x}_{i}$ and we define $A\left(x_{i}\right)=\left\{\widehat{y}_{i} \in \widehat{X}_{i}:\left[x_{i}, \widehat{y}_{i}\right] \in A\right\}$ and $A\left(\widehat{x}_{i}\right)=\left\{y_{i} \in X_{i}\right.$ : $\left.\left[y_{i}, \widehat{x}_{i}\right] \in A\right\}$. If $A_{i} \subset X_{i}$ and $\widehat{A}_{i} \subset \widehat{X}_{i}, A_{i} \otimes \widehat{A}_{i}$ denotes the set $\left\{\left[y_{i}, \widehat{y}_{i}\right] \in X: y_{i} \in\right.$ $A_{i}$ and $\left.\widehat{y}_{i} \in \widehat{A}_{i}\right\}$.

We shall give an application of a fixed point theorem to an intersection theorem as follows:

THEOREM 3. Let $\left\{X_{i}\right\}_{i \in I}$ be a family of non-empty convex sets, each in a locally convex Hausdorff topological vector space $E_{i}$. For each $i \in I$, let $D_{i}$ be a non-empty compact subset of $X_{i}$. Suppose that $\left\{A_{i}\right\}_{i \in I},\left\{B_{i}\right\}_{i \in I}$ are two families of subsets of $X=\prod_{i \in I} X_{i}$ having the following properties:

(1) for each $i \in I$ and $x_{i} \in D_{i}$, the set $B_{i}\left(x_{i}\right)$ is open in $\widehat{X}_{i}$,

(2) for each $i \in I$, and $\widehat{y}_{i} \in \widehat{X}_{i}$, the set $B_{i}\left(\widehat{y}_{i}\right) \cap D_{i}\left(=\left\{x_{i} \in D_{i}:\left[x_{i}, \widehat{y}_{i}\right] \in B_{i}\right\}\right)$ $\neq \emptyset$ and $\operatorname{co}\left(B_{i}\left(\widehat{y}_{i}\right) \cap D_{i}\right) \subset A_{i}\left(\widehat{y}_{i}\right) \cap D_{i}$.

Then we have $\bigcap_{i \in I} A_{i} \neq \emptyset$.

Proof: Define $S_{i}, T_{i}: X \rightarrow 2^{D_{i}}$ as follows :

$$
\begin{aligned}
& S_{i}(y)=B_{i}\left(\widehat{y}_{i}\right) \cap D_{i}, \\
& T_{i}(y)=A_{i}\left(\widehat{y}_{i}\right) \cap D_{i}, \quad \text { for each } y \in X .
\end{aligned}
$$


Then by (2), for each $i \in I$ and $y \in X, \operatorname{co} S_{i}(y) \subset T_{i}(y)$ and $S_{i}(y) \neq \emptyset$. By (1), for each $i \in I$ and $x_{i} \in D_{i}$,

$$
\begin{aligned}
S_{i}^{-1}\left(x_{i}\right) & =\left\{y \in X: x_{i} \in S_{i}(y)\right\} \\
& =\left\{y \in X: x_{i} \in B_{i}\left(\widehat{y}_{i}\right) \cap D_{i}\right\}\left(=\left\{y \in X: x_{i} \in B_{i}\left(\widehat{y}_{i}\right)\right\}\right) \\
& =\left\{y \in X:\left[x_{i}, \widehat{y}_{i}\right] \in B_{i}\right\} \\
& =X_{i} \otimes B_{i}\left(x_{i}\right)
\end{aligned}
$$

is open in $X$.

By Theorem 2, there exists $x \in D=\prod_{i \in I} D_{i}$ such that $x \in T(x)=\prod_{i \in I} T_{i}(x)$, that is, $x_{i} \in A_{i}\left(\widehat{x}_{i}\right)$ for all $i \in I$ and hence $x=\left[x_{i}, \widehat{x}_{i}\right] \in \bigcap_{i \in I} A_{i}$. Therefore $\bigcap_{i \in I} A_{i} \neq \emptyset$. This
completes the proof.

We remark that Theorem 3 is closely related to but not comparable to Theorem 2 of Shih-Tan [12] which was a non-compact generalisation of Fan's intersection theorem [6] (in our case, the space $\boldsymbol{E}_{\boldsymbol{i}}$ is required to be locally convex).

Next we shall give two equilibrium existence theorems for a non-compact abstract economy with an infinite number of commodities and an infinite number of agents. We first give some definitions in equilibrium theory. Let the set $I$ of agents be any (possibly uncountable) set. An abstract economy $\Gamma=\left(X_{i}, A_{i}, B_{i}, P_{i}\right)_{i \in I}$ is defined as a family of ordered quaduples $\left(X_{i}, A_{i}, B_{i}, P_{i}\right)$ where $A_{i}, B_{i}: \prod_{j \in I} X_{j} \rightarrow 2^{X_{i}}$ are constraint correspondences and $P_{i}: \prod_{j \in I} X_{j} \rightarrow 2^{X_{i}}$ is a preference correspondence. An equilibrium for $\Gamma$ is a point $\widehat{x} \in X=\prod_{i \in I} X_{i}$ such that for each $i \in I, \widehat{x}_{i} \in$ $c l_{X_{i}} B_{i}(\widehat{x})$ and $A_{i}(\widehat{x}) \cap P_{i}(\widehat{x})=\emptyset$. When $A_{i}=B_{i}$ for each $i \in I$, our definitions of an abstract economy and an equilibrium coincide with the standard definitions, for example in Borglin-Keiding [1, p.315] or in Yannelis-Prabhakar [15, p.242].

We shall first show that by applying Himmelberg's fixed point theorem [8, Theorem 2] instead of Ky Fan's fixed point theorem [5], the proof of Theorem 6.1 of YannelisPrabhakar [15] can be used to prove its non-compact case.

Theorem 4. Let $\Gamma=\left(X_{i}, A_{i}, B_{i}, P_{i}\right)_{i \in I}$ be an abstract economy such that for each $i \in I$,

(1) $X_{i}$ is a non-empty convex subset of a locally convex Hausdorff topological vector space $E_{i}$ and $D_{i}$ is a non-empty compact subset of $X_{i}$,

(2) for each $x \in X=\prod_{i \in I} X_{i}, A_{i}(x)$ is non-empty, $A_{i}(x) \subset B_{i}(x) \subset D_{i}$ and $B_{i}(x)$ is convex,

(3) the correspondence $c l B_{i}: X \rightarrow 2^{X_{i}}$ defined by $\left(\operatorname{cl} B_{i}\right)(x)=c l_{X_{i}} B_{i}(x)$ for each $x \in X$, is upper semicontinuous, 
(4) for each $y \in D_{i}, A_{i}^{-1}(y)$ is open in $X$,

(5) for each $y \in X_{i}, \quad P_{i}^{-1}(y)$ is open in $X$,

(6) for each $x \in X, \quad x_{i} \notin \operatorname{co} P_{i}(x)$,

(7) the set $\left\{x \in X: \operatorname{co} A_{i}(x) \cap \operatorname{co} P_{i}(x) \neq \emptyset\right\}$ is paracompact.

Then $\Gamma$ has an equilibrium $\widehat{x} \in X$, that is, for each $i \in I$,

$$
\widehat{x}_{i} \in c_{X_{i}} B_{i}(\widehat{x}) \quad \text { and } \quad A_{i}(\widehat{x}) \cap P_{i}(\widehat{x})=0
$$

Proof: We first fix $i \in I$. Define $\phi_{i}: X \rightarrow 2^{X_{i}}$ by

$$
\phi_{i}(x)=\operatorname{co} A_{i}(x) \cap \operatorname{co} P_{i}(x) \quad \text { for each } x \in X
$$

By (4), (5) and Lemma 5.1 of Yannelis-Prabhakar [15], it is easy to see that for each $y \in X_{i}, \phi_{i}^{-1}(y)$ is open in $X$. Let $U_{i}=\left\{x \in X: \phi_{i}(x) \neq \emptyset\right\}$. Since $U_{i}=$ $\bigcup_{y \in X_{i}} \phi_{i}^{-1}(y), U_{i}$ is open in $X$. By (7), $U_{i}$ is paracompact. Note that $\left.\phi_{i}\right|_{U_{i}}: U_{i} \rightarrow 2^{X_{i}}$ has the following properties :

(i) for each $x \in U_{i},\left.\phi_{i}\right|_{U_{i}}(x)$ is non-empty and convex,

(ii) for each $y \in X_{i},\left(\left.\phi_{i}\right|_{U_{i}}\right)^{-1}(y)=\phi_{i}^{-1}(y) \cap U_{i}$ is open in $U_{i}$.

By Theorem 3.1 of Yannelis-Prabhakar [15] (which is the case $S=T$ in our Theorem 1), there exists a continuous selection $f_{i}: U_{i} \rightarrow 2^{X_{i}}$ such that $f_{i}(x) \in \phi_{i} \mid U_{i}(x)$ for all $x \in U_{i}$. Define $F_{i}: X \rightarrow 2^{X_{i}}$ by

$$
F_{i}(x)= \begin{cases}\left\{f_{i}(x)\right\}, & \text { if } x \in U_{i}, \\ c l_{X_{i}} B_{i}(x), & \text { if } x \notin U_{i}\end{cases}
$$

By (3) and Lemma 6.1 of Yannelis-Prabhakar [15], $F_{i}: X \rightarrow 2^{X_{i}}$ is upper semicontinuous on $X$. Clearly for each $x \in X, F_{i}(x)$ is a non-empty closed convex subset of $D_{i}$ by (2). Finally we define $F: X \rightarrow 2^{X}$ by

$$
F(x)=\prod_{i \in I} F_{i}(x) \quad \text { for each } x \in X
$$

It follows from Lemma 3 of Fan [5] that $F$ is upper semicontinuous on $X$. Obviously for each $x \in X, F(x)$ is a closed convex subset of $D=\prod_{i \in I} D_{i}$. By Tychonoff's product theorem (for example see Dugundji $[4, \mathrm{p} .224]$ ), $D$ is a compact subset of $X$. Hence by Theorem 2 of Himmelberg [8], there exists a point $\widehat{x} \in D$ such that $\widehat{x} \in F(\widehat{x})$. If $\widehat{x} \in U_{i}$ for some $i \in I$, then $\widehat{x}_{i}=f_{i}(\widehat{x}) \in \operatorname{co} A_{i}(\widehat{x}) \cap \operatorname{co} P_{i}(\widehat{x}) \subset \operatorname{co} P_{i}(\widehat{x})$ which contradicts (6). Thus for each $i \in I$, we must have $\widehat{x} \notin U_{i}$ so that $\widehat{x}_{i} \in c_{X_{i}} B_{i}(\widehat{x})$ and 
$\operatorname{co} A_{i}(\widehat{x}) \cap \operatorname{co} P_{i}(\widehat{x})=\emptyset$. Consequently, $\widehat{x}$ is an equilibrium for $\Gamma$. This completes the proof.

As we have seen in the proof, we can obtain a stronger separation result, that is, for each $i \in I, \operatorname{co} A_{i}(\widehat{x}) \cap \operatorname{co} P_{i}(\widehat{x})=\emptyset$.

Theorem 4 generalises Theorem 6.1 of Yannelis-Prabhakar [15] in the following ways :

(i) for each $i \in I$, the space $E_{i}$ need not be metrisable,

(ii) for each $i \in I$, the set $X_{i}$ need not be compact, and

(iii) the set $I$ of agents need not be countable.

TheOREM 5. Let $\Gamma=\left(X_{i}, A_{i}, B_{i}, P_{i}\right)_{i \in I}$ be an abstract economy such that for each $i \in I$, the following conditions hold:

(1) $X_{i}$ is a non-empty convex subset of a locally convex Hausdorff topological vector space $E_{i}$ and $D_{i}$ be a non-empty compact subset of $X_{i}$,

(2) for each $x \in X, A_{i}(x)$ is non-empty and co $A_{i}(x) \subset B_{i}(x) \subset D_{i}$,

(3) for each $y_{i} \in D_{i}$, the set $\left[\left(c o P_{i}\right)^{-1}\left(y_{i}\right) \cup F_{i}\right] \cap A_{i}^{-1}\left(y_{i}\right)$ is open in $X$, where $F_{i}=\left\{x \in X: A_{i}(x) \cap P_{i}(x)=\emptyset\right\}$,

(4) for each $x \in X, x_{i} \notin \operatorname{co} P_{i}(x)$.

Then $\Gamma$ has an equilibrium.

Proof: For each $i \in I$, let $G_{i}=\left\{x \in X: A_{i}(x) \cap P_{i}(x) \neq \emptyset\right\}$ and for each $x \in X$, let $I(x)=\left\{i \in I: A_{i}(x) \cap P_{i}(x) \neq \emptyset\right\}$. For each $i \in I$, we define the correspondences $S_{i}, T_{i}: X=\prod_{i \in I} X_{i} \rightarrow 2^{D_{i}}$ by

$$
\begin{aligned}
& S_{i}(x)=\left\{\begin{array}{rr}
\operatorname{co} P_{i}(x) \cap A_{i}(x), & \text { if } i \in I(x), \\
A_{i}(x), & \text { if } i \notin I(x),
\end{array}\right. \\
& T_{i}(x)=\left\{\begin{array}{rr}
\operatorname{co} P_{i}(x) \cap B_{i}(x), & \text { if } i \in I(x), \\
B_{i}(x), & \text { if } i \notin I(x) .
\end{array}\right.
\end{aligned}
$$

Then we have the following properties:

(i) for each $i \in I$ and $x \in X$, co $S_{i}(x) \subset T_{i}(x)$ and $S_{i}(x) \neq \emptyset$,

(ii) for each $i \in I$ and $y_{i} \in D_{i}$,

$$
\begin{aligned}
S_{i}^{-1}\left(y_{i}\right) & =\left\{\left[\left(\operatorname{co} P_{i}\right)^{-1}\left(y_{i}\right) \cap A_{i}^{-1}\left(y_{i}\right)\right] \cap G_{i}\right\} \cup\left[A_{i}^{-1}\left(y_{i}\right) \cap F_{i}\right] \\
& =\left[\left(\operatorname{co} P_{i}\right)^{-1}\left(y_{i}\right) \cap A_{i}^{-1}\left(y_{i}\right)\right] \cup\left[A_{i}^{-1}\left(y_{i}\right) \cap F_{i}\right] \\
& =\left[\left(\operatorname{co} P_{i}\right)^{-1}\left(y_{i}\right) \cup F_{i}\right] \cap A_{i}^{-1}\left(y_{i}\right)
\end{aligned}
$$

is open in $X$ by (3). 
By Theorem 2, there exists $\widehat{x} \in D$ such that $\widehat{x}_{i} \in T_{i}(\widehat{x})$ for all $i \in I$. By (4) and the definition of $T_{i}$, we have $\widehat{x}_{i} \in B_{i}(\widehat{x})$ and $A_{i}(\widehat{x}) \cap P_{i}(\widehat{x})=\emptyset$ for all $i \in I$. This completes the proof.

Finally we remark that Theorems 4 and 5 are closely related to those results of Shafer-Sonnenschein [11, p.347], Borglin-Keiding [1, p.315] and Tarafdar [14, Theorem 3.1].

\section{REFERENCES}

[1] A. Borglin and H. Keiding, 'Existence of equilibrium actions and of equilibrium: a note on the 'new' existence theorem', J. Math. Econom. 3 (1976), 313-316.

[2] F.E. Browder, 'The fixed point theory of multi-valued mappings in topological vector spaces', Math. Ann. 177 (1968), 283-301.

[3] H.F. Cullen, Introduction to general topology (D. C. Heath, Boston, 1968).

[4] J. Dugundji, Topology (Allyn and Bacon, Inc., Boston, 1966).

[5] K. Fan, 'Fixed-point and minimax theorems in locally convex topological linear spaces', Proc. Nat. Acad. Sci. U.S.A. 38 (1952), 131-136.

[6] K. Fan, 'Sur un théorème minimax', C.R. Acad. Sci. Paris Ser. 1259 (1964), 3925-3928.

[7] K. Fan, 'A minimax inequality and applications', in Inequalities III, Editor O. Shisha (Academic Press, New York, 1972).

[8] C.J. Himmelberg, 'Fixed points of compact multifunctions', J. Math. Anal. Appl. 38 (1972), 205-207.

[9] M. Lassonde, 'Fixed point for Kakutani factorizable multifunctions', J. Math. Anal. Appl. $152(1990), 46-60$.

[10] E. Michael, 'A note on paracompact spaces', Proc. Amer. Math. Soc. 4 (1953), 831-838.

[11] W. Shafer and H. Sonnenschein, 'Equilibrium in abstract economies without ordered preferences', J. Math. Econom. 2 (1975), 345-348.

[12] M.H. Shih and K.-K. Tan, 'Non-compact sets with convex sections', Pacific J. Math. 119 (1985), 473-479.

[13] D.R. Smart, Fixed point theorems (Cambridge University Press, Cambridge, MA, 1974).

[14] E. Tarafdar, 'A fixed point theorem and equilibrium point of an abstract economy', $J$. Math. Econom. 20 (1991), 211-218.

[15] N.C. Yannelis and N.D. Prabhakar, 'Existence of maximal elements and equilibria in linear topological spaces', J. Math. Econom. 12 (1983), 233-245.

Department of Mathematics

Sichuan Normal University

Chengdu, Sichuan

China
Department of Mathematics Education Chungbuk National University

Cheongju 360-763

Korea

Department of Mathematics, Statistics and Computing Science

Dalhousie University

Halifax NS

Canada B3H 3J5 